\title{
Survival and the Art of Profit Maximization ${ }^{1}$
}

\author{
Prajit K. Dutta \\ Department of Economics \\ Columbia University \\ New York, NY 10027
}

\author{
Rangarajan K. Sundaram \\ Department of Finance \\ Stern School of Business \\ New York University \\ New York, NY 10012
}

April 3, 2001

Forthcoming: Review of Economic Design

\footnotetext{
${ }^{1}$ We would like to thank a number of people for helpful comments and suggestions including, especially, Jeff Banks, Ananth Madhavan, Kalyan Chatterjee, and an anonymous referee.
} 


\begin{abstract}
We study the relationship between rationality and economic survival in a simple dynamic model, where agents from different populations interact repeatedly through random matching. An explicit criterion ("bankruptcy") determines whether agents survive each interaction; all agents are presumed to be aware of this criterion. Survival in each interaction depends on two factors: the strategies agents adopt when they interact, and the wealth levels they bring to the game. The model is completely symmetric with the only difference between the agents of different populations being their objectives.

We consider the case where there are two populations of agents in which all agents from one group have as their objective, maximizing the expected profits from each interaction, while all agents from the other attempt simply to maximize the probability of surviving (i.e., not going bankrupt in) the interaction. We show that under the equilibrium dynamics, the proportions of each group in the total population must be bounded away from zero, but the balance is in favor of the survival-probability maximizers in that their numbers as a fraction of total population must always exceed $1 / 2$, and can be arbitrarily close to unity. On the other hand, the fraction of total wealth controlled by the expected profit maximizers must also be at least $1 / 2$, and can asymptotically tend to unity.
\end{abstract}




\section{Introduction}

This paper examines the link between rationality and economic survival in the context of a model where agents have differing objectives. Our inquiry is motivated by the question of whether, and in what sense, environments select agents who "adapt;" and the extent to which success in survival can be attributed to "rational" decision rules such as profit- or utility-maximization. This line of inquiry has, of course, a long history in economics. Our paper is related to at least two strands of the existing literature.

One, beginning with Alchian (1950) and Friedman (1953), adopts the common viewpoint that in a market selection mechanism, realized profitability is a necessary condition for survival. ${ }^{1}$ On the implication of this assumption for identifying the most "fit" decision rules, there has been less unanimity. Some authors, such as Friedman (1953) and Enke (1951), have argued that competitive forces would lead, in such a world, to the selection of firms that maximized profits. $^{2}$ When there is perfect knowledge regarding the consequences of different choices by the firms, this appears an uncontroversial assertion. In a world of uncertainty, however, where firms are, in effect, choosing probability distributions over profit levels, the notion of "profit maximization" lacks an a priori meaning, since the market selects firms whose realized (and not anticipated) profits are highest. This problem was pointed out by Alchian (1950), but he did not propose any one criterion as most "rational" or "fit" in such situations. Alchian notwithstanding, it would be fair to say that economic theory has subsequently, and rather uncritically, reinterpreted the Friedman argument as identifying the maximization of expected profits with fitness, and as the criterion to be adopted in the marketplace. ${ }^{3}$

One problem with this branch of the literature has been the absence of a formal model within which its conclusions may be examined. ${ }^{4}$ Certainly, the a priori connection between "rationality" and "expected profit maximization" is not obvious. In any reasonable economic model, firms must be assumed to have knowledge of the selection mechanism. A "rational" firm would presumably incorporate knowledge of this mechanism into its decision calculus, and there is no reason at all to expect, under these conditions, that the firm will choose expected-profit maximization as the "best" criterion. It is important to point out that giving firms knowledge of this selection mechanism, and allowing them to incorporate it into their decision process, creates no problems under certainty, for, here, maximizing profits and maximizing the probability of survival are (by

\footnotetext{
${ }^{1}$ For example, " ... those who realize positive profits are the survivors; those who suffer losses disappear." (Alchian, 1950, p.213)

${ }^{2}$ To quote Friedman (1953) "Unless the behavior of businessmen in some way approximated behavior consistent with the maximization of returns, it seems unlikely they would be in business for long."

${ }^{3}$ Undoubtedly, one reason behind the the popularity of the expected-utility (or expected-profit) maximization hypothesis lies in the analytical tractability of models in which all agents pursue such goals.

${ }^{4}$ However, see Blume and Easley (1988) and Dutta \& Radner (1999).
} 
definition!) the same.

A second strand of the literature, that on "evolutionary games," originating with Maynard Smith (1982), models more explicitly the interaction among alternative strategies within the context of a game and studies the long-run fitness of different strategies. A typical evolutionary game model ${ }^{5}$ has the following structure. The population of agents is divided into groups on the basis of the strategies the agents adopt, with all agents within a group playing the same strategy. Agents are then randomly matched. In each period, the group or groups whose strategies fare better than the population average are then assumed to grow in size (more precisely, their proportion in the population increases). The aim is then to examine whether there are strategies which are "evolutionarily stable," i.e, that survive this selection mechanism. This literature has resulted in important insights into selection mechanisms and the problems of obtaining "stable" strategies. As a theory of economic evolution, however, it suffers from at least three shortcomings. First, in much of this literature, agents' strategies are exogenously fixed: players do not actively seek to adapt or react to the strategy choices of those with whom they are matched ${ }^{6}$ In particular, they do not take cognizance of the survival mechanism into account when making their choices. Secondly, the rule determining survival is somewhat arbitrary. Third, there are no market features in these models (such as, say, market shares or wealth shares). Thus, while who survives is determined, the question of asymptotic control of the market, a logically different issue from which group's numbers are preponderant in the limit, cannot be examined.

Our aim in this paper is to study a simple dynamic model that is rich enough to admit the following important features:

1. There is an explicit survival criterion, and all agents are aware of this criterion.

2. There are different kinds of agents who interact with each other at regular intervals. Some agents simply try to maximize the probability of surviving each encounter, while others maximize the expected level of profits from the interactions.

3. Each member of each group is also characterized by his wealth level ${ }^{7}$ at any point in time, so that there are two dynamic processes that evolve together: the proportion of each type in the overall population, and the wealth share of each type in the overall wealth.

Our model will have the appealing feature that one group of agents-the survival-probability maximizers-will, ceteris paribus, have a greater probability of survival, but conditional on survival, the wealth level of the other group-the expected-profit maximizers-will be higher.

\footnotetext{
${ }^{5}$ See, e.g., Friedman (1988) or the Journal of Economic Theory Symposium Issue on Evolutionary Games (August 1992).

${ }^{6}$ Some models of evolutionary learning (e.g., Canning, 1990) provide agents with endogeneous decision-making power. Here, typically, agents' actions have to be best-responses to some conjectured population strategies.

${ }^{7}$ Wealth in our model is a proxy for market power.
} 
Our aim is then to track the performance of the two groups over time along two dimensions. First, how do the proportions of the two groups in the overall population change over time? Second, how do the proportions of total wealth controlled by each group change over time? We adopt the position at the outset that the "fitness" of a strategy in the marketplace must be measured along both dimensions. A strategy that leads to "survival" while getting swamped in growth by other strategies, is not "fit" in any sense in which economists would wish to use that word.

The details of the model we study borrows much from both branches of the literature discussed above. From the first, we adopt the notion of "bankruptcy" as determining the condition of failure; survival therefore entails avoiding bankruptcy. From the second, we import the idea of dividing the population into different types of agents, and the matching specification. More formally, we consider a world with large populations of two types of agents, who interact repeatedly over a number of "rounds." In any round, agents from each type are randomly matched into pairs. Each pair then plays a "bankruptcy" game, the outstanding feature of which is that not all risk can be hedged (there is real uncertainty). In this game, players pick actions that jointly determine the parameters of the stochastic process governing the growth rate and evolution of their wealth levels. The game terminates when one player has become bankrupt, i.e., when his wealth level reaches zero. The bankrupt agent then leaves the game, while the surviving agent takes his wealth level at this point into the next round.

Agents from the two populations are presumed to have different preferences. In particular, we assume that those of the first type choose their strategies to maximize their expected wealth level at the end of each round (in our model this is analogous to maximizing expected profits from each encounter); while agents from the second population simply maximize their probability of surviving (i.e., not going bankrupt in) each round.

Our main conclusions may be summarized as follows. First, when the environment is "symmetric" (either group faces exactly the same possibilities and payoffs, the only difference being their objectives), expected profit-maximizers never fare better than survival-probability maximizers on population share, and often do strictly worse. On the other hand, expected-profit maximizers fare at least as well as survival-probability maximizers on wealth share, and sometimes do strictly better. In particular, when the stage-game is "linear," they do as well on the wealth-share dimension, but strictly worse on the population share dimension. When the stage game is sufficiently non-linear, they do strictly better on wealth share, even though they do worse on pupulation share.

Secondly, symmetry is important for a fair comparison. If the model is asymmetric (i.e., the environment favors one group or the other), much depends on who has access to the superior technology. If the environment facors the expected-profit maximizers (no matter how slightly), they can come to dominate the market along both dimensions, in population-share as 
well as wealth-share; but exactly the opposite is true if the environment tilts towards the survivalprobability maximizers. Finally, if wealth generation is subject to increasing returns, then we find that expected-profit maximizers can come to control the entire market wealth even if their share in the population falls below $50 \%$.

The two papers closest to ours are Dutta and Radner (1999) and Blume and Easley (1988). The Dutta-Radner paper asks exactly the same question as the current one: (under what conditions) does a market whose selection mechanism is bankruptcy select an entrepreneur with profit-maximizing preferences? The conclusion reached by that paper is even more stark than the one we uncover here: in the Dutta-Radner model, every profit-maximizing entrepreneur goes bankrupt in finite time with probability one, but there are survivalists who survive forever with positive probability. Hence, after a long period of time, the proportion of the population that is maximizing profits is essentially zero. Moreover, the wealth share of profit-maximizing entrepreneurs also goes to zero.

The model in the Dutta-Radner paper differs from the current one in one important respect: unlike here, there is no product-market interaction in that model (although there is capital-market interaction since every firm has to come to a common capital market to get funded). Once funded, each firm is run independently —or equivalently, is run in a competitive product market. Hence the differential performance of profit-maximizers versus survivalists is driven not by relative differences in riskiness of choice (as in the current analysis) but rather by absolute differences. In particular, the key insight of the Dutta-Radner paper is that a profit-maximizing entrepreneur would never want to build up arbitrarily large cash reserves (since doing so compromises the profits that can be distributed to shareholders). On the other hand, a survivalist entrepreneur would adopt a dividend payout policy that allows for arbitrarily large reserves. From a long-term survival point of view, a cash reserve that is finite-sized must necessarily get run down to zero in finite time.

The Blume and Easley paper also investigates the "survival" of "profit-maximizing" agents. However, their model is a pure gambling model with no production and hence their agents are more analogous to consumers, some of whom pursue expected utility maximization while others do not. Moreover there is no bankruptcy in the model. Hence, the worst that can happen to an agent type is that their wealth share can decrease over time (although their population share, by definition, must remain unchanged). Blume and Easley show that under a variety of scenarios it is entirely possible that the wealth share of expected profit maximizers sink to zero. They were not able, however, to establish which behavioral rules would necessarily do better than the expected utility maximizers.

The remainder of this paper is organized as follows. Section 2 describes our model and motivates the stage-game structure that we use. Section 3 analyzes the "linear" game, both with and without symmetry. Section 4 completes the analysis by looking at non-linear extensions of the model. 


\section{The Evolutionary Game}

This section is divided into two parts. In subsection 2.1, we outline the basic structure of the evolutionary game that we study in this paper. Subsection 2.2 then presents the specific questions of interest.

\subsection{The Basic Structure}

We consider a model with two populations of agents, denoted I and II, each with a continuum of members. The initial measure of each population is normalized to unity. Members of either population are characterized at each point in time by their wealth levels. The initial wealth level of each member of each population is fixed at some $y>0$.

In each period $t=0,1,2, \ldots$, of an infinite horizon, agents of the two populations are matched into playing a symmetric stage game that we sometimes refer to as a "bankruptcy game." The action space for either player in this game is a non-degenerate compact set $K \subset \mathbb{R}$. These actions may be thought of essentially as constituting the players' choice of "risk levels" in the game; this interpretation of $K$ will become clear shortly. The outcome of the game decides two things: the player who "survives" the game, and the wealth level of the surviving player at the end of the game. This outcome, in turn, depends on two factors, viz., the actions the players take in the game, and the wealth levels they bring to the game. More formally, letting $k_{i}$ denote the action choice of the type- $i$ player, and $y_{i}$ that player's wealth level at the start of the game, the outcome of the game is determined by four functions $\left(\rho_{1}, \rho_{2}, h_{1}, h_{2}\right)$ where $\rho_{i}\left(y_{1}, y_{2} ; k_{1}, k_{2}\right)$ specifies the probability that player $i$ "survives" the game (i.e., does not go bankrupt), and $h_{i}\left(y_{1}, y_{2} ; k_{1}, k_{2}\right)$ specifies the terminal wealth level of player $i$ in the event that he survives. The bankrupt player (say $j$ ) then exits the game, while the survivor $i$ moves into the next round with the wealth level $h_{i}(\cdot)$.

Additional structure will be required of the functions $\rho_{i}$ and $h_{i}$ in the sequel. We will require the conditions A1-A4 listed below. The first two assumptions are symmetry conditions on the game. (On the importance of symmetry, see subsection 3.3 below.) The third assumption involves the natural condition that, ceteris paribus, the probability of success (as also the terminal wealth contingent on winning) is increasing in own wealth. Finally, Assumption A4 makes clear the interpretation of actions as choices of risk levels: it asserts that, ceteris paribus, a higher action results in a lower probability of survival, but, contingent on survival, also results in a higher wealth level. In conjunction with $\mathrm{A} 3$, this assumption creates the tension that forms an important component of our model: a, less risky action today implies a greater probability of survival this period (i.e., in the short run), but also results in a lower rate of growth of wealth, which, by A3, reduces long run survival prospects. 
A1 $\rho_{1}\left(y_{1}, y_{2} ; k_{1}, k_{2}\right)=\rho_{2}\left(y_{2}, y_{1} ; k_{2}, k_{1}\right)$.

A2 $h_{1}\left(y_{1}, y_{2} ; k_{1}, k_{2}\right)=h_{2}\left(y_{2}, y_{1}, k_{2}, k_{1}\right)$.

A3 For each $i$ and for all fixed $\left(y_{j} ; k_{1}, k_{2}\right)$, the functions $\rho_{i}$ and $h_{i}$ are increasing in $y_{i}$.

A4 For each $i$, and for all fixed $\left(y_{1}, y_{2} ; k_{j}\right), \rho_{i}$ is decreasing in $k_{i}$, while $h_{i}$ is increasing in $k_{i}$.

Returning to our description of the stage game, the players of population I (whom we will also refer to as "expected-profit maximizers") aim to maximize their expected profits from each interaction, i.e., to solve, given $k_{2}$

$$
\max \left\{\rho_{1}\left(y_{1}, y_{2}, k_{1}, k_{2}\right) \cdot h_{1}\left(y_{1}, y_{2}, k_{1}, k_{2}\right) \mid k_{1} \in K\right\}
$$

Members of the second population (whom we call the "survival probability maximizers"), on the other hand, have as their objective simply maximizing the probability of surviving each encounter. That is, they solve, given $k_{1}$,

$$
\max \left\{\rho_{2}\left(y_{1}, y_{2}, k_{1}, k_{2}\right) \mid k_{2} \in K\right\}
$$

Let $K_{1}\left(y_{1}, y_{2} ; k_{2}\right)$ and $K_{2}\left(y_{1}, y_{2} ; k_{1}\right)$ denote, respectively, the solutions to the optimization problems of players 1 and 2. As always, a (Nash) equilibrium of the game is a strategy pair which is such that no player can benefit from unilateral deviation, i.e., a, pair $\kappa\left(y_{1}, y_{2}\right)=$ $\left(\kappa_{1}\left(y_{1}, y_{2}\right), \kappa_{2}\left(y_{1}, y_{2}\right)\right)$, such that for each $\left(y_{1}, y_{2}\right)$, we have $\kappa_{1}\left(y_{1}, y_{2}\right) \in K_{1}\left[y_{1}, y_{2} ; \kappa_{2}\left(y_{1}, y_{2}\right)\right]$ and $\kappa_{2}\left(y_{1}, y_{2}\right) \in K_{2}\left[y_{1}, y_{2} ; \kappa_{1}\left(y_{1}, y_{2}\right)\right]$.

This completes the description of the stage game. The following subsection utilizes this structure to identify the questions of interest.

\subsection{Equilibrium Dynamics and Fitness}

Throughout the paper, to keep equilibrium dynamics tractable, we will assume that all members of a given population use the same strategy. Let $\kappa\left(y_{1}, y_{2}\right)$ be some equilibrium strategy pair. The selection $\kappa$ together with the initial (common) wealth level $y$ determines the evolution of per capita wealth levels of the survivors in each population as follows (here, as in the sequel, we suppress dependence of these objects on $\kappa$ and $y$ ): 


$$
\begin{array}{ll}
y_{i 0}=y & \\
k_{i t}=\kappa_{i}\left(y_{1 t}, y_{2 t}\right), & t=0,1,2, \ldots \\
y_{i t}=h_{i}\left(y_{1 t-1}, y_{2 t-1}, k_{1 t-1}, k_{2 t-1}\right), & t=1,2, \ldots
\end{array}
$$

The conditional probability $\rho_{i t}$ of a type- $i$ player surviving into period- $(t+1)$ given survival upto period-t is also determined:

$$
\rho_{i t}=\rho_{i}\left(y_{1 t}, y_{2 t}, k_{1 t}, k_{2 t}\right), \quad t=0,1,2 \ldots
$$

Identifying the probability of a member of a population surviving with the fraction of that population which survives, the fraction $p_{i t}$ of population $i$ which survives into period $t$ is:

$$
p_{i t}=\prod_{\tau=0}^{t-1} \rho_{i \tau}
$$

Thus, the relative share $p_{t+1}$ of population I in the total population at time $(t+1)$ (viz., the ratio of population I to population II at that point) is:

$$
p_{t+1}=\frac{p_{1 t+1}}{p_{2 t+1}}=\left(\frac{\rho_{1 t}}{\rho_{2 t}}\right) p_{t}
$$

while the ratio $s_{t}$ of the wealth controlled by population I to that controlled by population II is:

$$
s_{t}=\frac{p_{1 t} y_{1 t}}{p_{2 t} y_{2 t}}=\left(\frac{y_{1 t}}{y_{2 t}}\right) p_{t}
$$

The sequences $p_{t}$ and $s_{t}$ form the focus of our study in this paper. To state the precise questions of interest requires a little more notation.

Let $P_{\infty}$ and $S_{\infty}$ denote the set of all limit points of the sequences $\left\{p_{t}\right\}$ and $\left\{s_{t}\right\}$, respectively. Note that the sequences $\left\{p_{t}\right\}$ and $\left\{s_{t}\right\}$ (as also, therefore, $P_{\infty}$ and $S_{\infty}$ ) depend on the equilibrium strategy pair $\kappa($.$) . We will sometimes highlight this dependence by writing P_{\infty}(\kappa), S_{\infty}(\kappa)$, etc.

A strategy $\kappa_{1}$, for population I will be called a fit strategy against $\kappa_{2}$, if it is the case that either $\inf P_{\infty}\left(\kappa_{1}, \kappa_{2}\right)>0$, or $\inf S_{\infty}\left(\kappa_{1}, \kappa_{2}\right)>0$. This definition of fitness is really a minimal one: a strategy is fit as long as it does not lead to total failure. (By our symmetry assumptions, fit strategies always exist-every strategy is fit against itself.)

As a stronger definition, a strategy $\kappa_{1}$, for population I will be called a most fit strategy against $\kappa_{2}$ if the following condition holds: there exists no other strategy $\widehat{\kappa}_{1}$ such that $\widehat{p} \geq p$ 
and $\widehat{s} \geq s$ for all $\widehat{p} \in P_{\infty}\left(\widehat{\kappa}_{1}, \kappa_{2}\right), p \in P_{\infty}\left(\kappa_{1}, \kappa_{2}\right), \widehat{s} \in S_{\infty}\left(\widehat{\kappa}_{1}, \kappa_{2}\right)$, and $s \in S_{\infty}\left(\kappa_{1}, \kappa_{2}\right)$, with at least one strict inequality for some $(\widehat{p}, p)$ or $(\widehat{s}, s)$. In words, $\kappa_{1}$ is most fit against $\kappa_{2}$, if there is no other strategy for Population I all of whose limit points dominate all of those under $\left(\kappa_{1}, \kappa_{2}\right)$, with strict inequality somewhere.

Finally, the strategy $\kappa_{1}$, will be termed an unbeatable strategy for Population I, if it is a most fit strategy against any strategy $\kappa_{2}$ for Population II.

Analogous definitions of "fit," "most fit," and "unbeatable" strategies hold for population II. We note the important point that (once again, by the symmetry assumptions), the sets of fit, most fit, and unbeatable strategies for the two populations coincide. So, for instance, a strategy $\kappa_{i}$ is a fit strategy for Population $i$ against a strategy $\kappa_{j}$ for population $j$ if, and only if, $\kappa_{i}$ is a fit strategy for Population $j$ when Population $i$ adopts $\kappa_{j}$.

Of course, the fitness of a strategy confers no equilibrium properties on it whatsoever; in particular, it can be the case that $\left(\kappa_{1}, \kappa_{2}\right)$ are a, pair of strategies that are fit against each other (or most fit against each other, or even unbeatable), but that do not constitute a Nash equilibrium pair.

Indeed, our focus in this paper is primarily on the relationship between fitness and equilibrium. Specifically, we are interested in the following questions. When is it the case that equilibrium strategy profiles are also fit (or most fit or unbeatable) against each other? In particular, when is this true for Population I (the expected profit maximizers)? Conversely, when do most fit strategies (and, especially, unbeatable ones) constitute an equilibrium? Finally, when is it the case that, in equilibrium, one side comes to dominate the market from the wealth angle $\left(S_{\infty}=\{\infty\}\right)$ or the population angle $\left(P_{\infty}=\{\infty\}\right)$ ?

\section{A Linear Model}

In this section, we present a first analysis of the evolutionary game of section 3 , by considering some restrictions on the functions $\rho_{i}$ and $h_{i}$. These restrictions arise out of a simple extension to a two-person setting of the much-studied framework of one-person bankruptcy problems. We detail this procedure in subsection 3.1 below. We term the model of the evolutionary game thus obtained the linear model, in part because the functions $h_{i}$ and $\rho_{i} h_{i}$ depend linearly on $y_{1}$ and $y_{2}$. In section 3.2, we then analyze the consequent evolutionary game dynamics. Finally, subsection 3.3 discusses the consequences of introducing asymmetry into the underlying model. 


\subsection{The Bankruptcy Game}

A vast literature in statistics and probability (and also economics) has examined the following one-person bankruptcy problem (see, e.g., Karatzas and Shreve (1988), or Dubins and Savage (1957). An agent begins with a wealth level of $y>0$. This wealth evolves according to the process

$$
d y=m d t+v^{1 / 2} d W_{t}
$$

where $W$ is standard Brownian motion. The process terminates and the agent fails at the first $T$ at which $y(T)=0$. The agent is presumed to have some control over this process (say, over the instantaneous drift $m$ or the variance $v$ ), and selects his strategy to maximize some desired objective, such as the expected time to failure.

A two-person version of this problem, that can be viewed as a symmetric bankruptcy game in which players' actions simultaneously determine (a) the probability of bankruptcy of each player, and (b) the wealth level of the non-bankrupt player at the time of the other's bankruptcy, may be obtained by modifying this problem as follows. Let $K \subset \mathbb{R}$ be a non-degenerate compact set with lower endpoint $\min K$ and upper endpoint $\max K$. ( $K$ represents the players' (common) choice of actions in the game.) Let $g: K \times K \rightarrow \mathbb{R}_{++}$be any continuous and symmetric function that is strictly increasing in its first argument and strictly decreasing in the second.

Suppose now that player 1 's wealth level $y_{1}$ evolves according to some exogenous process as above:

$$
d y_{1}=v^{1 / 2} d W_{t}
$$

(for purposes of symmetry, we have set $m$ to zero), while given the action choices $\left(k_{1}, k_{2}\right)$ for the players, player 2's wealth level evolves according to

$$
d y_{1}=-g\left(k_{1}, k_{2}\right) d y_{2}
$$

That is, each dollar lost by player 2 results in a gain of $\$ g\left(k_{1}, k_{2}\right)$ to player 1 , while each dollar lost by player 1 results in a gain of $\$\left[1 / g\left(k_{1}, k_{2}\right)\right]$ to player 2 . Assume that $g$ is multiplicatively symmetric, i.e., $g\left(k_{1}, k_{2}\right) \cdot g\left(k_{2}, k_{1}\right)=1$ for all $\left(k_{1}, k_{2}\right) \in K \times K^{8}$. The game terminates at the first $T$ (if any) at which some player becomes bankrupt, i.e., at the first $T$ at which $\min \left\{y_{1}(T), y_{2}(T)\right\}=0$.

Simple calculations yield the following. Fix an action pair $\left(k_{1}, k_{2}\right)$. Let $\left(y_{1}, y_{2}\right)$ represent the players' initial wealth levels at the start of the bankruptcy game. Then, given $\left(y_{1}, y_{2}, k_{1}, k_{2}\right)$, the

\footnotetext{
${ }^{8}$ Note that in this case we have $g(k, k)=1$ for all $k \in K$.
} 
terminal wealth level of player 1 , contingent on player 2 going bankrupt, is:

$$
h_{1}\left(y_{1}, y_{2}, k_{1}, k_{2}\right)=y_{1}+g\left(k_{1}, k_{2}\right) y_{2}
$$

while the corresponding figure for player 2 is:

$$
h_{2}\left(y_{1}, y_{2}, k_{1}, k_{2}\right)=y_{2}+y_{1} / g\left(k_{1}, k_{2}\right)
$$

Note that player $i$ 's wealth level remains between 0 and $h_{i}\left(y_{1}, y_{2}, k_{1}, k_{2}\right)$ as long as the bankruptcy game is being played; the moment $i$ 's wealth level hits zero, he is bankrupt. Conversely, $j$ goes bankrupt if $i$ 's wealth level reaches $h_{i}\left(y_{1}, y_{2}, k_{1}, k_{2}\right)$. From basic stochastic calculus, it follows that the probability of hitting one or the other boundary is one; and that the probability that player $i(=1,2)$ survives (i.e., that player $j \neq i$ goes bankrupt) is:

$$
\rho_{i}\left(y_{1}, y_{2}, k_{1}, k_{2}\right)=\frac{y_{i}}{h_{i}\left(y_{1}, y_{2}, k_{1}, k_{2}\right)}
$$

These values for the terminal wealth and survival probabilities yield a natural interpretation of the action levels $k_{i}$ as choices of "risk levels" given our assumptions on $g$ : ceteris paribus, the terminal wealth $h_{i}$ that will result contingent on success increases with $k_{i}$, but so also does the probability of failure $\rho_{i}$. In particular, assumption A4 is met. It is simple to verify that so also are assumptions $\mathrm{A} 1-\mathrm{A} 3$.

The functions $h_{1}, h_{2}, \rho_{1}, \rho_{2}$, as given by (10)-(12), together with the monotonicity restrictions on $g$, complete the description of the stage game. We now turn to the implications of this structure for the evolutionary game of section 2 .

\subsection{Equilibrium and its Properties}

We begin with a description of the equilibria of the stage game. The following result, that establishes the existence of (a multitude of) equilibria in this game, is an immediate consequence of the properties of $g$ and the forms of the functions $\rho_{i}$ and $h_{i}$ :

Proposition 3.1 For any $\left(y_{1}, y_{2}, k_{1}, k_{2}\right)$, we have $K_{1}\left(y_{1}, y_{2} ; k_{2}\right)=K$, and $K_{2}\left(y_{1}, y_{2} ; k_{1}\right)=$ $\{\min K\}$. Thus, given any $\left(y_{1}, y_{2}\right)$, the pair $\left(k_{1}, k_{2}\right) \in K \times K$ constitutes an equilibrium at $\left(y_{1}, y_{2}\right)$ if, and only if, $k_{2}=\min K$.

Proof For any fixed $\left(y_{1}, y_{2} ; k_{1}, k_{2}\right),(10)$ and (12) imply $\rho_{1} h_{1}=y_{1}$, which is independent of $k_{1}$. Thus, any $k_{1} \in K$ is a best-response for the player from population I to any $k_{2}$ at any $\left(y_{1}, y_{2}\right)$. That $K_{2}\left(y_{1}, y_{2} ; k_{1}\right)=\{\min K\}$ follows immediately from the fact that $\rho_{2}$ is decreasing in $k_{2}$. 
Thus, any equilibrium selection $\kappa\left(y_{1}, y_{2}\right)$ must have $\kappa_{2}\left(y_{1}, y_{2}\right)=\min K$, but $\kappa_{1}\left(y_{1}, y_{2}\right)$ is completely unrestricted. Nonetheless, there is enough structure in the model to completely characterize equilibrium dynamics. Fix any equilibrium selection $\kappa($.$) . Recall that p_{t}$ denotes the period- $t$ ratio of the population of type 1's to that of type 2's, while $s_{t}$ is the period$t$ ratio of the wealth controlled by type 1's to that controlled by type 2's. Recall also that $P_{\infty} \subset \mathbb{R}_{+}$and $S_{\infty} \subset \mathbb{R}_{+}$denote the set of limit points of the respective sequences. Let $\bar{g}=g(\max K, \min K)>1$. We will prove the following:

Proposition 3.2 In any equilibrium $\kappa$, we have $S_{\infty}=\{1\}$, and $P_{\infty} \subset[1 / \bar{g}, 1]$.

Proof Let $\left(y_{1 t}, y_{2 t}\right)$ denote the sequence of per capita wealth levels from $y$ under $\kappa$, and let $\left(k_{1 t}, \min K\right)$ denote the corresponding sequence of actions. For notational ease, also denote by $g_{t}$ the term $g\left(k_{1 t}, \min K\right)$.

Then, $y_{10}=y=y_{20}$, while for $t \geq 0$, we have $y_{1 t+1}=y_{1 t}+g_{t} y_{2 t}$, and $y_{2 t+1}=y_{2 t}+y_{1 t} / g_{t}$. It follows that for all $t \geq 0$,

$$
\frac{y_{1 t+1}}{y_{2 t+1}}=g_{t}
$$

Now note that, by substituting from (10) and (11) into (12), we obtain

$$
\frac{\rho_{1}\left(y_{1}, y_{2} ; k_{1}, k_{2}\right)}{\rho_{2}\left(y_{1}, y_{2} ; k_{1}, k_{2}\right)}=\frac{y_{1}}{g\left(k_{1}, k_{2}\right) y_{2}}
$$

Therefore, $\rho_{10} / \rho_{20}=1 / g_{0}$, while for $t \geq 1$,

$$
\frac{\rho_{1 t}}{\rho_{2 t}}=\frac{y_{1 t}}{g_{t} y_{2 t}}=\frac{g_{t-1}}{g_{t}}
$$

Using the recursive definition of the $p_{t}$ sequence from (5), we obtain, as a consequence, for $t \geq 0$ :

$$
p_{t+1}=\frac{g_{t-1}}{g_{t}} \frac{g_{t-2}}{g_{t-1}} \ldots \frac{g_{0}}{g_{1}} \frac{1}{g_{0}}=\frac{1}{g_{t}} .
$$

It follows immediately that for any $t \geq 0$,

$$
s_{t+1}=\left(\frac{y_{1 t+1}}{y_{2 t+1}}\right) p_{t+1}=g_{t} \frac{1}{g_{t}}=1
$$

establishing one part of the result.

The other part is a simple consequence of the relation above that $p_{1 t+1} / p_{2 t+1}=1 / g t$ : since $k_{2 t}=\min K$ for all $t$, we have 


$$
g\left(k_{1 t}, k_{2 t}\right) \in[g(\min K, \min K), g(\max K, \min K)]=[1, \bar{g}]
$$

Thus, the set of limit points of the sequence $\left\{1 / g_{t}\right\}$ (which is $P_{\infty}$ ) is contained in $[1 / \bar{g}, 1]$.

Proposition 3.2 establishes that in this simple model, the expected profit maximizers cannot come to dominate the market on either front, when they are faced with agents who simply maximize the short-run probability of survival. Indeed, more is true. Since $S_{\infty}=1$, and $P_{\infty} \leq$ $1 / 2$, many (in fact, in some sense, most $^{9}$ ) equilibrium strategies for Population I are not even most fit strategies:

Proposition 3.3 An equilibrium strategy $\kappa_{1}$ for Population I is a most fit strategy against the strategy $\kappa_{2}$ specified by $\kappa_{2}\left(y_{1}, y_{2}\right)=\min K$ for all $\left(y_{1}, y_{2}\right)$ if, and only if, the resulting sequence $k_{1 t}$ satisfies $\lim _{t \rightarrow \infty} k_{1 t}=\min K$.

Proof If Population I also plays the strategy $\kappa_{2}$, we will have $g_{t}=g(\min K, \min K)$ for all $t$, resulting in $P_{\infty}=S_{\infty}=1$. If Population I plays any strategy $\kappa_{1}$ such that, under $\left(\kappa_{1}, \kappa_{2}\right)$, $k_{1 t} \nrightarrow \min K$, then, along some subsequence, we must have $g_{t}$ converging to a limit $\gamma>1$. Then, $1 / \gamma \in P_{\infty}\left(\kappa_{1}, \kappa_{2}\right)$, so $\kappa_{2}$ dominates $\kappa_{1}$ against $\kappa_{2}$, so $\kappa_{1}$ cannot be a most fit strategy.

Remark It is easy to see that given any pair of strategies $\left(\kappa_{1}, \kappa_{2}\right)$ in this model, we must have $S_{\infty}=1$. Thus, the fitness of one strategy against another must be measured only along the lines of how limit population proportions are affected. It is thus immediate that $\kappa_{1}\left(y_{1}, y_{2}\right) \equiv \min K$ is an unbeatable strategy for either population.

To sum up, in the model considered in this subsection, the total wealth of the expected profit maximizers never grows fast enough for them to come to dominate the market on the wealth front, while their population shares, when they play the survival probability maximizers, is bounded above by $1 / 2$. Their equilibrium strategies are "typically" not most-fit strategies against their opponents' strategies, except in exceptional circumstances such as the conditions outlined in Proposition 3.3. (This is true even if they are not playing survival probability maximizers, obviously.) On the other hand, it is the case that the unique equilibrium strategy for the survival probability maximizers is also an "unbeatable" strategy.

\subsection{A Remark on the Importance of Symmetry}

A number of assumptions guaranteed symmetry in the game considered above, including the conditions that the wealth process of player 1 has zero drift, and that the odds-function $g$ is

\footnotetext{
${ }^{9}$ Recall that any strategy is an equilibrium strategy for player 1
} 
symmetric. The purpose of this subsection is to point out that if these symmetry assumptions are weakened, the conclusions of the model could change dramatically, since a systematic bias now enters the model.

We illustrate this process by considering a slight modification in the background stochastic process that was used to motivate the bankruptcy game. To elaborate, suppose, instead of (8), the evolution of player 1 's wealth level is given by

$$
d y_{1}=m d t+v^{1 / 2} d W_{t}
$$

where the drift $m$ is not necessarily zero. The rest of the structure is exactly as in subsection 3.1 ; in particular, we continue assuming that the wealth levels of players 1 and 2 are related by

$$
d y_{1}=-g\left(k_{1}, k_{2}\right) d y_{2}
$$

where $g$ has the same properties as in subsection 3.1. Note that $m$ could be as close to zero as desired, so we could be very "close" to the model of subsections 3.1-3.2.

Standard arguments from stochastic calculus show that when $m>0$, the probability $\rho_{1}\left(y_{1}, y_{2} ; k_{1}, k_{2}\right)$ of player 1 going bankrupt first, given $\left(y_{1}, y_{2} ; k_{1}, k_{2}\right)$, is given by:

$$
\rho_{1}\left(y_{1}, y_{2} ; k_{1}, k_{2}\right)=\frac{1-e^{-\lambda y_{1}}}{1-e^{-\lambda\left(y_{1}+g\left(k_{1}, k_{2}\right) y_{2}\right)}}
$$

where $\lambda=2 m / v$; while in the case $m<0$, we have:

$$
\rho_{1}\left(y_{1}, y_{2} ; k_{1}, k_{2}\right)=\frac{e^{\theta y_{1}}-1}{e^{\theta\left(y_{1}+g\left(k_{1}, k_{2}\right) y_{2}\right)}-1}
$$

where $\theta=-2 m / v$. The functions $h_{1}$, and $h_{2}$ remain unaltered from subsection 3.1 ; and as there, we again have $\rho_{2}=1-\rho_{1}$.

Note that for $i=1,2, h_{i}$ is increasing in $k_{i}$, while $\rho_{i}$ is decreasing in $k_{i}$. Thus, the actions $k_{i}$ continue to represent levels of risk: higher returns require accepting a lower probability of survival. Also, it is still the case that $h_{i}$ is increasing in $y_{i}$. Thus, assumptions A3 and A4 are met, and since the functions $h_{i}$ have not changed, so also is A2. Assumption Al is however violated, since the probabilities of survival are no longer symmetric.

The following result establishes that, in contrast to the zero drift case, the set of equilibrium strategies when $m \neq 0$ is quite small.

Proposition 3.4 When $m>0$, the unique equilibrium action pair at any $\left(y_{1}, y_{2}\right)$ is given by $\left(k_{1}, k_{2}\right)=(\max K, \min K)$. When $m<0$, the unique equilibrium, action pair at any $\left(y_{1}, y_{2}\right)$ is given by $k_{1}=k_{2}=\min K$. 
Proof We prove the Proposition for the case $m>0$. The case in $m<0$ is handled analogously. Fix any $\left(y_{1}, y_{2}\right)$. First consider player 1 's response problem for fixed $k_{2} \in K$ :

$$
\max _{k_{1} \in K}\left(\frac{1-e^{-\lambda y_{1}}}{1-e^{-\lambda\left(y_{1}+g\left(k_{1}, k_{2}\right) y_{2}\right)}}\right)\left(y_{1}+g\left(k_{1}, k_{2}\right) y_{2}\right)
$$

This is equivalent to solving

$$
\min _{k_{1} \in K}\left(\frac{1-e^{-\lambda\left(y_{1}+g\left(k_{1}, k_{2}\right) y_{2}\right)}}{y_{1}+g\left(k_{1}, k_{2}\right) y_{2}}\right)
$$

Since $\left(1-e^{-\lambda x}\right)$ is a concave function of $x$, the ratio $\left(1-e^{-\lambda x}\right) / x$ declines in $x$. Thus, minimizing (23) requires maximizing the denominator, or selecting $k_{1}=\max K$.

That player 2 always wishes to play $\min K$ follows simply from the fact that $\rho_{2}$ is decreasing in $k_{2}$.

More interestingly, we can also show that equilibrium dynamics are now determinate as regards both population- and wealth-shares; indeed, they are not only determinate, they also result in extreme predictions regarding limit population- and wealth-shares that depend only on whether $m$ is positive or negative. Namely, if $m>0$ (i.e., the drift favors the expected profit maximizers), then in the limit this population must both (a) control all the wealth and (b) be preponderant in numbers in the overall population; while if $m<0$, a similar conclusion is true of the survival probability maximizers:

Proposition 3.5 If $m>0$, then in the unique equilibrium, $P_{\infty}=S_{\infty}=\{+\infty\}$, while if $m<0$, then in the unique equilibrium, $P_{\infty}=S_{\infty}=\{0\}$.

Proof For notational ease, let $\bar{g}=g(\max K, \min K)$. Denote by $\left(y_{1 t}(m), y_{2 t}(m)\right)$ the resulting sequence of equilibrium wealth levels when the drift is $m$. We have for all $t \geq 0$,

$$
\begin{aligned}
& y_{1 t+1}(m)=y_{1 t}(m)+g_{t}(m) y_{2 t}(m) \\
& y_{2 t+1}(m)=y_{2 t}(m)+y_{1 t}(m) / g_{t}(m)
\end{aligned}
$$

where $g_{t}(m)=g(m)=\bar{g}$ if $m>0$, and $g_{t}(m)=g(m)=1$ if $m<0$. It is trivial therefore that in both cases, we have for all $t \geq 1, y_{1 t+1}(m)=2 y_{1 t}(m)$, and $y_{2 t+1}(m)=2 y_{2 t}(m)$. Thus, we obtain

$$
\begin{aligned}
& y_{1 t+1}(m)=2^{t}(1+g(m)) y \\
& y_{2 t+1}(m)=2^{t}(1+1 / g(m)) y
\end{aligned}
$$

In particular, we have $y_{1 t}(m) / y_{2 t}(m)=g(m)$ for all $t \geq 1$. 
First, suppose $m>0$. We suppress dependence on $m$. Using $y_{1 t}+g_{t} y_{2 t}=y_{1 t+1}=2 y_{1 t}$ in (19), we obtain

$$
\frac{\rho_{1 t}}{\rho_{2 t}}=\frac{1-e^{-\lambda y_{1 t}}}{e^{-\lambda y_{1 t}}-e^{-2 \lambda y_{1 t}}}=e^{\lambda y_{1 t}}
$$

Therefore,

$$
p_{t+1}=\prod_{\tau=0}^{t} \frac{\rho_{1 t}}{\rho_{2 t}}=e^{\lambda \sum y_{1 \tau}}=e^{\left(2^{t}-1\right) \lambda(1+\bar{g}) y},
$$

and since $2^{t} \rightarrow \infty$ as $t \rightarrow \infty, p_{t+1} \rightarrow \infty$, establishing one part of the result.

To see the other part, note that $y_{1 t} / y_{2 t}=\bar{g}$ for all $t \geq 1$. So,

$$
s_{t+1}=\frac{p_{1 t+1} y_{1 t+1}}{p_{2 t+1} y_{2 t+1}}=p_{t+1} \frac{y_{1 t+1}}{y_{2 t+1}}=p_{t+1} \bar{g},
$$

and $s_{t}$ also goes to infinity with $t$.

The results for $m<0$ are established analogously, and are omitted.

\section{A Generalization of the Linear Model}

The analysis of the previous section appears to depend on the "linearity" of the underlying structure. It is tempting to think that if $h_{1}$ or $\rho_{1} h_{1}$, were to depend non-linearly on (say were convex in) $y_{1}$, the result that $S_{\infty}=\{1\}$ could not arise, and, in fact, with sufficient convexity, one might even obtain $S_{\infty}=\{+\infty\}$. In this section, we show, using a simple generalization of the linear model, that this intuition is partly correct.

So let $\alpha \in \mathbb{R}_{++}$. We will consider a family of models parametrized by $\alpha$, in which the case of $\alpha=1$ corresponds to the linear model. As in the linear model, let $g\left(k_{1}, k_{2}\right)$ be any function on $K \times K$, which is strictly increasing in $k_{1}$ and strictly decreasing in $k_{2}$. We begin by replacing the definitions of $h_{i}$ given by (10)-(11) with

$$
h_{1}\left(y_{1}, y_{2} ; k_{1}, k_{2}\right)=\left(y_{1}^{\alpha}+g\left(k_{1}, k_{2}\right) y_{2}^{\alpha}\right)^{1 / \alpha}
$$

and

$$
h_{2}\left(y_{1}, y_{2} ; k_{1}, k_{2}\right)=\left(y_{1}^{\alpha} / g\left(k_{1}, k_{2}\right)+y_{2}^{\alpha}\right)^{1 / \alpha}
$$

Secondly, we replace the definition of the functions $\rho_{i}$ given by (12) with

$$
\rho_{1}\left(y_{1}, y_{2} ; k_{1}, k_{2}\right)=\frac{y_{1}^{\alpha}}{y_{1}^{\alpha}+g\left(k_{1}, k_{2}\right) y_{2}^{\alpha}}
$$


and

$$
\rho_{2}\left(y_{1}, y_{2} ; k_{1}, k_{2}\right)=\frac{g\left(k_{1}, k_{2}\right) y_{2}^{\alpha}}{y_{1}^{\alpha}+g\left(k_{1}, k_{2}\right) y_{2}^{\alpha}}
$$

Note that when $\alpha=1$, this model coincides with the linear model. We begin our analysis with the following:

Proposition 4.1 If $\alpha<1$, the unique stage game equilibrium strategies at any $\left(y_{1}, y_{2}\right)$ are $\left(k_{1}, k_{2}\right)=(\max K, \min K)$, while if $\alpha>1$, the unique equilibrium strategies at any $\left(y_{1}, y_{2}\right)$ are $\left(k_{1}, k_{2}\right)=(\min K, \min K)$.

Proof Consider first player 1 's best-response to $k_{2}$ at some $\left(y_{1}, y_{2}\right)$. Player 1 solves

$$
\max \left\{y_{1}^{\alpha}\left[y_{1}^{\alpha}+g\left(k_{1}, k_{2}\right) y_{2}^{\alpha}\right]^{\frac{1-\alpha}{\alpha}} \mid k_{1} \in K\right\}
$$

When $\alpha<1$, then $1-\alpha>0$, and the maximand is increasing in $k_{1}$, since $g$ is. Thus, $k_{1}=\max K$ is the unique solution here. If, on the other hand, $\alpha>1$, then the maximand is decreasing in $k_{1}$, and the unique solution is $k_{1}=\min K$.

That player 2's optimal strategy is to always play $\min K$ follows simply from the fact that $\rho_{2}$ is decreasing in $k_{2}$.

Proposition 4.2 If $\alpha<1$, then $S_{\infty}=\left\{\bar{g}^{(1-\alpha) / \alpha}\right\}>1$, and $P_{\infty}=\{1 / \bar{g}\}<1$, where $\bar{g}=$ $g(\max K, \min K)$. If $\alpha>1$, then $S_{\infty}=P_{\infty}=\{1\}$.

Proof First consider the case $\alpha<1$. At any $\left(y_{1}, y_{2}\right)$, we have,

$$
\frac{\rho_{1}\left(y_{1}, y_{2} ; \max K, \min K\right)}{\rho_{2}\left(y_{1}, y_{2} ; \max K, \min K\right)}=\frac{1}{\bar{g}}\left(\frac{y_{1}}{y_{2}}\right)^{\alpha}
$$

while

$$
\frac{h_{1}\left(y_{1}, y_{2} ; \max K, \min K\right)}{h_{2}\left(y_{1}, y_{2} ; \max K, \min K\right)}=(\bar{g})^{1 / \alpha}
$$

Thus, for $t \geq 1$, we have

$$
\frac{\rho_{1 t}}{\rho_{2 t}}=\frac{1}{\bar{g}}\left(\frac{y_{1 t}}{y_{2 t}}\right)^{\alpha}=\frac{1}{\bar{g}}\left(\frac{h_{1 t-1}}{h_{2 t_{1}}}\right)^{\alpha}=1
$$

This means that for $t \geq 1$,

$$
p_{t+1}=\frac{\rho_{1 t}}{\rho_{2 t}} p_{t}=p_{t}
$$


while for $t=1, p_{t}=\rho_{10} / \rho_{20}=1 / \bar{g}$, so $P_{\infty}=\{1 / \bar{g}\}$.

Moreover, we also have for $t \geq 1$

$$
s_{t+1}=p_{t+1} \frac{y_{1 t+1}}{y_{2 t+1}}=p_{t+1} \frac{h_{1 t}}{h_{2 t}}=\bar{g}^{(1-\alpha) / \alpha}
$$

This completes the proof for the case $\alpha<1$. When $\alpha>1$, then, in equilibrium, $g\left(k_{1}, k_{2}\right) \equiv 1$, and it is easy to see from this that we must have $p_{t}=s_{t}=1$ for all $t$.

Proposition 4.2 suggests that expected profit maximization can be a good thing in general: if $\alpha<1$, then members of population 1 fare no worse along either the population or wealth dimension than members of population 2, while if $\alpha>1$, they come to control more than $50 \%$ of the aggregate wealth in the limit. Indeed, this proportion increases towards $100 \%$ as $\alpha \downarrow 0$, even as their fraction of the population share remains bounded away from zero.

From a technical standpoint, it is easy to see what drives these results. When $\alpha<1$, then Proposition 4.1 establishes that the equilibrium strategy profile is $(\max K, \min K)$, so $\bar{g}=$ $g(\max K, \min K)>1$. Moreover, conditional on survival, player 1 will have a higher wealth level than player 2 , so we have $y_{1}>y_{2}$ in every period after the first. When these conditions are used in the definitions (24) and (26), a simple picture emerges. As $\alpha<1$ decreases towards zero, the payoff $h_{1}$ contingent on survival increases indefinitely, but while the survival probability decreases monotonically, it remains bounded above zero. Thus, lower values of $\alpha$ aid wealth accumulation more than they reduce survival probability, and this helps members of population 1 .

Ultimately, what these results - and those of the previous section-show is that much depends on the precise specification of the environment, but expected profit maximization generally does well overall. Expected profit maximization involves more risky strategies than merely seeking to stay alive. If this increased risk is more than compensated for in terms of wealth accumulation (which occurs when $\alpha<1$ ), then expected profit maximizers can not only be expected to survive, they will even come to dominate from a wealth standpoint. If the compensation is merely adequate (as happens when $\alpha=1$ ), then expected profit maximizers can only hold their own from a wealthshare standpoint. Finally, if the compensation is less than adequate $(\alpha>1)$, then expected profit maxiization involves an in-built defense-the equilibrium strategy profile changes, ensuring that wealth and population shares never fall below their initial levels.

\section{Conclusions}

Conventional economic theory makes frequent use of the hypothesis that firms seek to maximize expected profits, even though under uncertainty it is realized, and not anticipated, profits that 
determine a firm's survival. In this paper, we look at the justifiability of this hypothesis in an evolutionary sense using a simple dynamic framework. The trade-off captured by our model is that expected profit maximization involves strategies that are more risky than merely seeking to survive to the next period; however, contingent on survival, they lead to greater wealth accumulation which in turn improves future survival prospects.

We employ two criteria to judge the efficacy of expected profit maximization strategies: the fraction of the limit population who are expected profit maximizers, and the fraction of the aggregate wealth controlled by this segment. Overall, our results are mixed. From a population share standpoint, we find that expected profit maximization does not always do well: the limiting fraction of expected profit maximizers may be small. However, from the (perhaps more important) wealth share standpoint, they never do badly, and in some cases even come to dominate along this dimension. This suggests that the typical appeal to expected profit maximization may be justified at some level.

Balancing this, our results also indicate that the predictions of the model are sometimes quite sensitive to the precise specification of the environment, in some cases perhaps more sensitive than is comfortable. Thus, one cannot lightly extrapolate our results to a reality which is far more complex than in our model. In the final analysis, our results suggest that while there is reason for some optimism that the expected profit maximization may prove "fit" from an evolutionary standpoint, much reasearch remains to be done. 


\section{References}

[1] A. Alchian (1950) Uncertainty, Evolution, and Economic Theory, Journal of Political Economy $58,211-221$.

[2] Blume, L. and D. Easley (1992) Evolution and Market Behavior, Journal of Economic Theory 58(I), 9-40.

[3] Dutta, Prajit K. and Roy Radner (1999) Profit Maximization and the Market Selection Hypothesis Review of Economic Studies 66, 769-98.

[4] Enke, (1951) On Maximizing Profits: A Discussion between Chamberlin and Robinson, American Economic Review 41, 566-578.

[5] Friedman, D. (1988) Evolutionary Games: An Introduction for Economists, mimeo., University of California, Santa Cruz.

[6] Friedman, M. (1953) Essays in Positive Economics, University of Chicago Press, Chicago.

[7] Journal of Economic Theory: Symposium Issue on Evolutionary Game Theory Vol. 57(2), August 1992.

[8] Karatzas, I. and S. Shreve (1987) Brownian Motion and Stochastic Calculus, SpringerVerlag, Heidelberg.

[9] Maynard-Smith, J. (1982) Evolution and the Theory of Games, Cambridge University Press, Cambridge. 